\title{
Co-existence of Linear Lichen Planus and Zosteriform Vitiligo- Re-Exploration of Neurogenic Theory
}

\author{
Narasimhan $\mathrm{H}^{*}$ \\ SN medical College and Mathur das Mathur hospital, India
}

*Corresponding author: Hemamalini Narasimhan, SN medical College and Mathur das Mathur hospital, India, Tel: 8754763537; Email: jinney_17@hotmail.com

\section{Hypothesis}

Volume 4 Issue 4

Received Date: September 24, 2019

Published Date: October 28, 2019

DOI: $10.23880 /$ cdoaj-16000191

\section{Abstract}

Neuropeptides released due to stress can, as a result of oxidative damage, cause cytotoxicity to melanocytes and keratinlcyte. But the Blaschkoid pattern of two different auto immune diseases existing in the same patient at different sites is skeptical. Hence we revise the neurogenic theory and propose a new conjecture through a case report.

\section{Introduction}

An autoimmune disease occurring together has been a matter of debate for many years, some calling it a mere random probability [1]. Occurrence of DLE, psoriasis and vitiligo all in one patient is theoretically possible and rare, but no clear explanation has so far been described for certain patterns of diseases [2].

Studies have mentioned the potential of the vitiliginous sites to change antigen detection of CD 4 cells thereby changing target antigens. This explains why vitiligo patches can have lichen planus. Gavic, et al. proposed anxiety, depression and stress as causation in patients with both LP and vitiligo therefore could be the supplier of target antigens [3-5]. Hence, the emphasis has shifted from autoimmune theory to neural theory for special presentation as in our case because studies by $\mathrm{Wu}$, et al. mention the role of sympathetic nervous system for antibody against melanin production [6].

We are revisiting the neurogenic theory of vitiligo since Blaschko's line is just embryonic lines of skin and has no relationship to nerves and vessels. Hence exploring the mysterious relationship between diseases and it's patterns will lead to major management changes [4].

\section{Case Report}

A 23-year-old male presented with history of a pruritic polygonal papules with violaceous hue linearly with blaschkoid distribution over the left arm for last 3 weeks. The area around the linear lichenoid lesion had vitiliginous macule.

The patient was a known case of chronic stable segmental vitiligo, having well-demarcated depigmented macule of vitiligo in his right arm. Hair, scalp, nails, and mucosae were otherwise completely normal.

No similar family, drug/allergy history, occupational risks or dental procedures, photoexposure, other chronic comorbidities, history of autoimmune disease in the patient or his family. Differential diagnosis: linear Lichen planus, lichenoid dermatitis and drug induced LP.

Histology showed features were favoring a diagnosis of lichen planus. He was prescribed clobetasol propionate 
ointment which he applied once daily for 4 weeks to the lesion and was asked to follow up.

\section{Discussion}

Here the coexistence of linear lichen planus and zosteriform vitiligo is discussed as it is the first case to be reported and for finding the causal relationship between the pattern of disease $[7,8]$. Kachhawa D, et al. concluded that vitiliginous sites and sun exposed sites were both confounding factors for actinic Lichen planus [9].

As we know so many theories contribute to vitiligo and neurogenic theory sets well for segmental pattern but the reason for which is so far been debated. Neurogenic theory explains that the neuropeptides, CGRP, Catecholamine released due to stress or nerve damage causes oxidative damage and cytotoxicity [10].

Hypothesis: Since both melanocytes and nerves were derived from the neural crest cells, they carry similar antigens [11] and that blaschko lines are only connected with autonomic nervous system, we propose the following conjecture. If the target antigen was a neurotransmitter, sharing common haptens with melanocytes and keratinocytes which have undergone acquired genetic mosaicism, the cytotoxicity spreads along blaschko lines as the antibody affects the target ANS neurotransmitter and other mimicking antigens $[7,12]$.

This "common neurotransmitter antigen" theory explains the Blaschkoid distribution of autoimmune diseases and their existence at different sites because the target antigen can be chosen along any segment of skin and antibodies can attack keratinocytes and melanocytes at one segment to cause lichenoid pattern and simply melanocytes in another segment causing vitiligo.

This neurogenic theory is by far superior to other theories in explaining that two auto immune diseases can occur at different sites independent of each other. Further molecular studies are necessary to confirm the conjecture.

\section{References}

1. López-Jornet P, Parra-Perez F, Pons-Fuster A (2014) Association of autoimmune diseases with oral lichen planus: a cross-sectional, clinical study. J Eur Acad Dermatol Venereol 28(7): 895-899.
2. Zuzanna Ślebioda, Barbara Dorocka-Bobkowska, Elżbieta Szponar (2014) Coexistence of oral lichen planus and vitiligo-Case report Article. Dent Med Probl 51(4): 541-545.

3. Asarch A, Gottlieb AB, Lee J, Masterpol KS, Scheinman PL, et al. (2009) Lichen planus-like eruptions: an emerging side effect of tumor necrosis factor-alpha antagonists. J Am Acad Dermatol 61(1):104-111.

4. Asarch R (1996) 'Zosteriform' Lichen planus: Is it Zosteriform? Dermatology 192: 385-386.

5. Gavic L, Cigic L, Biocina Lukenda D, Gruden V, Gruden Pokupec JS (2014) The role of anxiety, depression, and psychological stress on the clinical status of recurrent aphthous stomatitis and oral lichen planus. J Oral Pathol Med 43(6): 410-417.

6. Wu CS, Yu HS, Chang HR, Yu CL, Yu CL, et al. 2(000) Cutaneous blood flow and adrenoceptor response increase in segmental-type vitiligo lesions. J Dermatol Sci 23(1): 53-62.

7. Peters EM, Handjiski B, Kuhlmei A, Hagen E, Bielas H, et al. (2004) Neurogenic inflammation in stressinduced termination of murine hair growth is promoted by nerve growth factor. Am J Pathol 165: 259-271.

8. David V, Georgios K, Sian H, Christopher B (2015) A Rare Colocalization of Lichen Planus and Vitiligo. Case Reports in Dermatological Medicine.

9. Ahmed K, Kachhawa D, Khullar R (1992) Coexistence of vitiligo and actinic lichen planus. Ind J Dermatol Venereol Leprol 58: 128-130.

10. Morrone A, Picardo M, de Luca C, Terminali O, Passi S, et al. (1992) Catecholamines and vitiligo. Pigment Cell Res 5(2): 65-69.

11. Orecchia GE (2000) Neural pathogenesis. In: Hann S, Nordlund J (Eds), Vitiligo. Oxford: Blackwell Science Ltd.

12. Bolognia JL, Orlow SJ, Glick SA (1994) Lines of Blaschko. J Am Acad Dermatol 31: 157-190. 